\title{
Age, senile dementia and ventricular enlargement
}

\author{
B M HU B B ARD A N J M ANDERSON
}

From the Department of Pathology, Ninewells Hospital and Medical School, Dundee, Scotland

\begin{abstract}
SUMMARY Necropsy measurements of cerebral ventricular volume and pericerebral space in senile dementia patients and age-matched controls indicate that ventricular enlargement is not an accurate diagnostic marker for cerebral atrophy. Furthermore, ventricles are of normal size for age in about $40 \%$ of all senile dementia patients including those with Alzheimer's disease.
\end{abstract}

The clinical diagnosis of Alzheimer type senile dementia (SDAT), the most frequent cause of intellectual deterioration in old age ${ }^{12}$ rests with the exclusion of other causes of organic dementia. A definite diagnosis is usually achieved only at necropsy for brain biopsy is seldom performed, but SDAT may be inferred if cerebral atrophy can be shown radiologically. Computed tomography (CT) has provided a safe non-invasive means of examining the size of the cerebral ventricles ${ }^{3}{ }^{4}$ and has been advocated as a screening procedure for elderly demented patients to find those without cerebral atrophy, some of whom may have treatable conditions such as hypothyroidism or pernicious anaemia, ${ }^{5}$ drug intoxication, benign cerebral neoplasms or psychiatric disturbances other than dementia. ${ }^{6}$ However, it remains uncertain how accurately ventricular enlargement in old age and senile dementia reflects cerebral atrophy since direct assessment of the pericerebral space by CT is technically difficult. Furthermore, on pathological examination some SDAT brains show comparatively little atrophy ${ }^{2} 7$ or have selective atrophy of the temporal lobes ${ }^{8}$ which does not affect the body of the ventricles so that a proportion of false negative results might be predicted if the diagnosis of SDAT were based on CT measurement of the main part of the cerebral ventricular system.

It was the aim of this morphometric investigation to examine the range of sizes of the cerebral ventricular system occurring in elderly persons, some severely demented during life and others non-demented, and to find how closely ventricular

Address for reprint requests: Dr JM Anderson, Ninewells Hospital, Ninewells, Dundee DD1 9SY, Scotland.

Accepted 5 May 1981 enlargement is associated with other pathological changes of SDAT.

\section{Material and methods}

The volume of the cerebral ventricles was measured in brains from 55 routine necropsy cases. Twentynine (nine males, 20 females, aged 64-92 years) had been severely demented, but none had affective disorders or schizophrenia; 27 were from long-stay psychogeriatric wards and two were awaiting admission. The 26 controls were from general medical and surgical wards and were selected after careful scrutiny of case records including nursing notes; no patient who had abnormal neurological signs or mental illness was included. Twenty-two of the controls (11 males, 11 females) were aged 65-95 years; four younger subjects (two males, two females, aged $23,38,42$ and 44 years) were also examined.

Brain volume was determined by displacement of $0.15 \mathrm{~N}$ saline from an Archimedes vessel. To avoid distortion of the ventricles, brains were suspended by a thread under the basilar artery during fixation in $15 \%$ formalin for 4 to 5 weeks. The cerebral hemispheres were cut into 25-30 coronal slices using an electrical slicing machine at a constant setting of $7 \mathrm{~mm}$. The volume proportion of the ventricular system was found by point-counting the brain slices on the Delesse principle ${ }^{9}$ using a cellulose acetate sheet with points at the angles of equilateral triangles of side $10 \mathrm{~mm}$. The absolute volume of the cerebral ventricles was then derived from the following formula:

$$
\frac{\text { Cerebral ventricular volume }=}{\text { To of points over the ventricles }} \times \frac{\text { Cerebral weight }}{\begin{array}{c}
\text { Specific gravity } \\
\text { of fixed brain }
\end{array}}
$$

A minimum of 1500 points was counted over each brain so that the percentage standard error incurred was $12-18 \%$ depending on the size of the ventricles. 
This was deemed an acceptable error in view of the large range (11 to $99 \mathrm{ml}$ ) of ventricular sizes in this investigation; it compares favourably with the estimated $20 \%$ error in the measurement of ventricular volume by CT scan. ${ }^{10}$ The effect of fixation on the volume of the brain was relatively small (mean inirease $3.3 \%$ ) and post-mortem delay did not significantly affect the mean ventricular size (mean value for controls $<24 \mathrm{~h}$ postmortem $24.0 \mathrm{ml}$, 24-48 h $36.0 \mathrm{ml}$; dements $<24 \mathrm{~h}$ postmortem $36.0 \mathrm{ml}, 24-48 \mathrm{~h}$ $48.0 \mathrm{ml}$ ) and so no corrections were attempted.

In 41 cases the cranial cavity volume was measured by the method of Davis and Wright.11 In this procedure a rubber balloon is placed in the skull cavity after removal of the brain, dura of the vault, falx and tentorium. The calvarium is held in place by a coronet clamp. The balloon is inflated with water to a pressure of $150 \mathrm{mmHg}$, tied off and removed. The water content corresponds to the internal volume of the skull. The difference between the cranial cavity volume and the fresh brain volume is referred to as the pericerebral space; a ratio of the pericerebral space to the cranial capacity is taken as a measure of brain atrophy since cranial cavity volume remains unchanged during life.11 Similarly, a ratio of the volume of the temporal lobes, found by point-counting brain slices as described above, to the cranial capacity gives a measure of temporal lobe atrophy.

For the diagnosis of SDAT the percentage of neurones bearing neurofibrillary tangles was determined using Ball's ${ }^{12}$ counting method on the subiculum of the hippocampal gyrus, an area of predilection for Alzheimer changes. ${ }^{13}$ A tangle count was preferred to measurement of senile plaques for it has been shown that hippocampal neurofibrillary change discriminates better between age change and Alzheimer senile dementia. ${ }^{2} 714$ Twenty-three dements had more than $5 \%$ of tangled neurones and were classified as SDAT. Of the six other dementia patients, one showed the selective temporal lobe sclerosis of Pick's disease and two had multiple microinfarcts indicating arteriopathic damage. Three cases remain undiagnosed.

\section{Results}

The control values showed a modest but significant rise in ventricular volume with age (fig 1); an exponential curve gave the best fit $(r=0.63$ $\mathrm{p}<0.001)$ which suggests that there is normally an acceleration of ventricular enlargement in old age (cerebral ventricular volume/brain volume $\%=$ $\left.0.633 \mathrm{e}^{0.017 \mathrm{age}}\right)$. By comparison, ventricular volume was normal or less than normal in nine $(31 \%)$ of the dementia patients, three $(10 \%)$ were close to the upper limit of normal, and just $17(59 \%)$ were well above the upper limit. Of the 23 dements showing histological changes of SDAT only 13 $(57 \%)$ had a ventricular volume above the upper limit of normal.
Comparison of the pericerebral space with ventricular size (fig 2) showed a linear correlation $(r=0.45 p<0.01)$ but this was not so close as

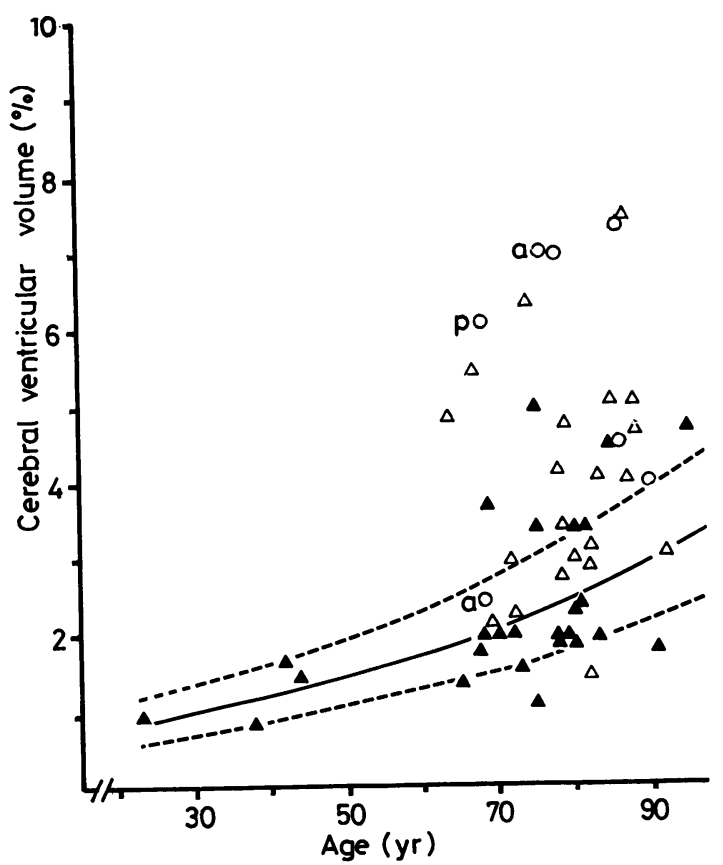

Fig 1 Plot of cerebral ventricular volume $(\%$ of brain volume) against age showing an exponential increase in controls ( ). The broken lines show the normal limits of the control range. In SDAT $(\triangle)$ and in other types of senile dementia $(O)$ about one-third of patients have normal-sized ventricles. $a=$ arteriopathic dementia; $p=$ Pick's disease.

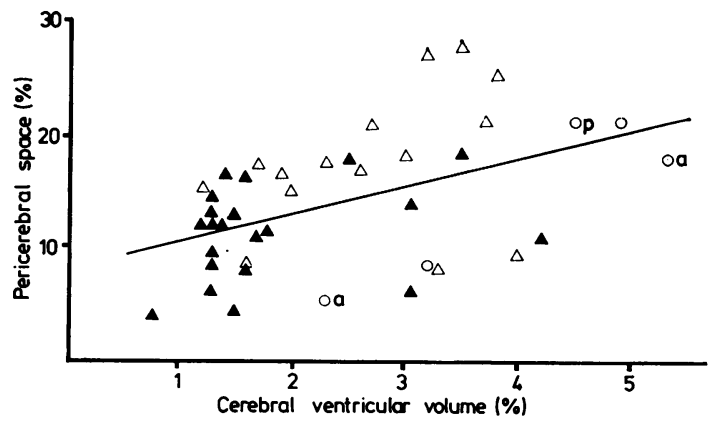

Fig 2 Plot of the pericerebral space expressed as a proportion of the cranial capacity (an index of cerebral atrophy) against cerebral ventricular volume showing a linear correlation but not close enough to suggest that ventricular enlargement is entirely due to cerebral atrophy $(r=0.45)$. $a=$ arteriopathic dementia; $p=$ Pick's disease. (Symbols as fig 1.) 
might be expected if ventricular enlargement in old age and senile dementia was entirely due to cerebral atrophy. The disparity between cerebral atrophy and ventricular enlargement is further emphasised by fig 3 which shows that ventricles

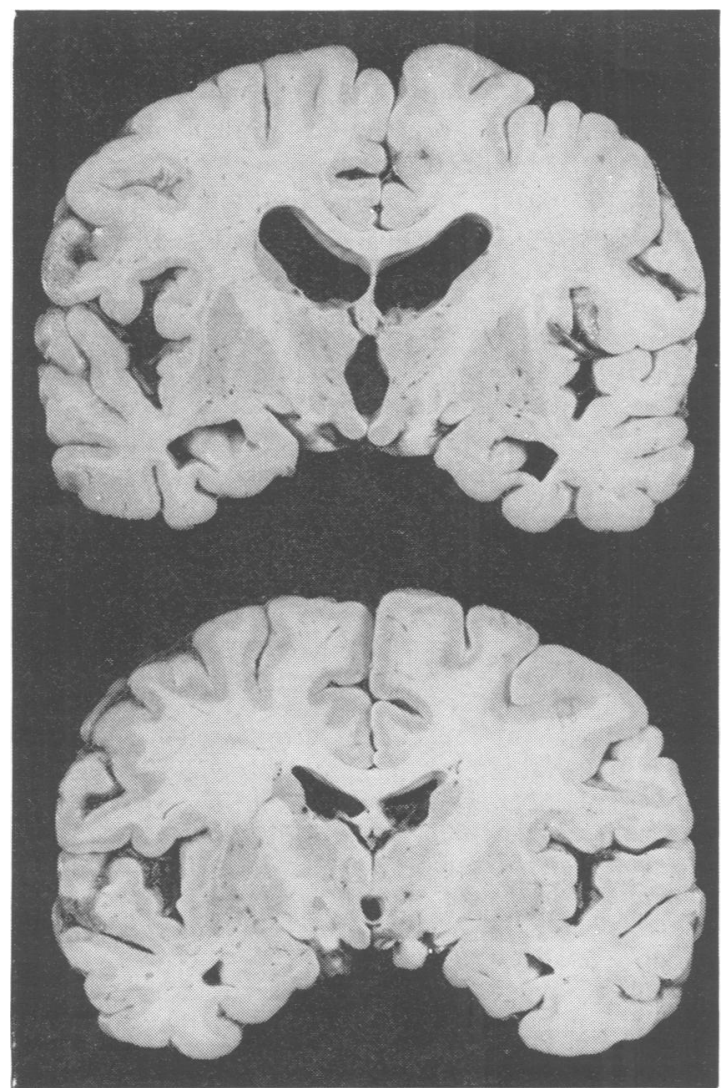

Fig 3 Cerebral atrophy is advanced in both of these SDAT brains (note the wide Sylvian fissures) but only one shows ventricular enlargement.

may be large or small in the presence of cerebral atrophy. In addition, temporal lobe atrophy was not accurately reflected by ventricular enlargement. Although 15 out of 20 dements had a reduced temporal lobe volume (that is temporal lobe volume/cranial capacity ratio $<9 \%$; mean control value for persons aged over $65=10 \cdot 1 \%$ ) this did not correlate closely with ventricular size $(r=0.39)$.

\section{Discussion}

In a necropsy study of cerebral ventricular volume it is important to consider the effect of postmortem decay for CT scans indicate that the ventricles become smaller after death. ${ }^{15}$ In our subjects over 65 years of age the upper limit of normal for the ventricular volume expressed as a percentage of the brain volume was about $5 \%$ whereas it is between $10 \%{ }^{16}$ and $14 \%$ when calculated from CT scans in life. This difference probably reflects the shift of cerebro-spinal fluid into the brain tissue after death. Experiments have shown that equilibration of brain and cerebro-spinal water and electrolytes occurs mainly in the first 12 hours after death with only minor changes after 24 hours $^{18}$ and sequential CT scans confirm that much of the ventricular contraction has taken place by 5 to 6 hours postmortem. ${ }^{15}$ The data in our investigation actually suggest some increase in ventricular size with extended postmortem interval, although this is not statistically significant and is probably fortuitous. Because only one necropsy was carried out less than 12 hours after the patient's death, we have thought it reasonable to assume that post-mortem changes were comparable throughout.

A ratio of the volume of the ventricles to the volume of the brain was used because this takes account of the normal biological variation in brain size although it may exaggerate the effects of cerebral atrophy. This ratio was employed since the range of normal brain size is considerably greater than the variable loss in brain mass through atrophy. Analysis of the absolute ventricular volumes did not alter the results significantly but, as might be expected, the values were more widely scattered, particularly those of the controls.

Our work reaffirms that there is an age-related increase in ventricular size as do recent investigations by CT scan. ${ }^{16} 17$ Early claims that ventricular enlargement occurs normally in old age have not been generally accepted because these were based on air encephalography which is performed only on persons under investigation for neurological disorders. ${ }^{17}$ Furthermore, a previous pathological enquiry found little difference in ventricular size between non-demented old people and younger age groups. ${ }^{19}$ However, the exponential type of increase in ventricular size with age suggested by our study and also by CT scan measurements $^{17}$ is consistent with the similar decline in brain volume during old age described by Davis and Wright. ${ }^{11}$ CT scans are carried out on many more patients than were formerly investigated by air encephalography, and a larger proportion are probably free from nervous disease, but the criticism can still be made that these CT observations $^{1617}$ and also our necropsy study relate to 
hospital patients who may not be representative of the normal population. Recently, Jacoby et $a l^{20}$ examined 50 psychiatrically and neurologically healthy volunteers from the community to establish genuine normative standards for CT scans in the elderly; they found only a non-significant trend to larger ventricles with age, but this may be due to the lack of any persons under the age of 62 years in their survey.

In our series pathological ventricular enlargement was by no means a reliable marker for senile dementia. No more than $59 \%$ of the dements had a definite increase in ventricular size and at most 13 out of 25 patients with SDAT showed this feature (fig 1). This is a much smaller proportion than was suggested by Gosling ${ }^{21}$ who found enlarged ventricles in $86 \%$ of dements compared with $11 \%$ of non-demented patients investigated for neurological disorders, but it should be noted that these patients were mainly in the sixth decade of life and therefore Gosling's findings are not entirely relevant to senile dementia, for cerebral atrophy tends to be more severe in pre-senile dementia. ${ }^{22}$ Our results are more in keeping with two CT scan investigations specifically concerned with old people which found very variable ventricular dimensions in senile dementia and a large overlap with the size range in nondemented persons. ${ }^{5}{ }^{23}$

The volume of the cerebral ventricles and of the pericerebral space were not so closely related $(r=0.45)$ as to suggest that ventricular enlargement in old age and senile dementia is entirely a consequence of cerebral atrophy. This finding has the obvious implication that CT observations on ventricle size cannot be taken as a measure of the degree of cortical atrophy. In particular, ventricular enlargement is unlikely to be of value as a sign of pathological cerebral atrophy in persons over the age of 80 years because senile dementia patients at this age tend to have selective temporal lobe atrophy ${ }^{8}$ and we have found no useful correlation between temporal lobe shrinkage and the size of the ventricular system. This conclusion is closely similar to that reached by Jacoby and Levy ${ }^{23}$ who found a low level of correlation between $\mathrm{CT}$ scan features of cortical atrophy and ventricular size in elderly control subjects $(r=0.38)$ and virtually no correlation in senile dements $(r=0 \cdot 16)$. These authors also reported that dementia patients over the age of 80 years less frequently showed ventricular enlargement than those below this age. Clearly a CT scan measurement of the pericerebral space might be more useful, but at present this is difficult to do with accuracy. The unreliability of CT scan measurements of pericerebral space and sulcal width may account for the absence of a clear relationship between these and the degree of intellectual impairment in old people. ${ }^{24}$ It is notable that Jacoby and Levy ${ }^{23}$ found a subjective assessment of the degree of cortical atrophy to discriminate better between senile dements and elderly controls than either of two objective measurements of ventricular dimensions. It is therefore to be hoped that technical refinement of CT will ultimately make measurement of the pericerebral space a routine matter and so obviate the need for clinical observations on ventricular enlargement in the context of cerebral atrophy.

We thank Professor J Swanson Beck for helpful criticism of the manuscript, Mr SA Ogston for advice on statistics, Mr RSR Fawkes for preparing the illustrations and Mrs I Bloomer for secretarial assistance. The work was supported by a grant from the Scottish Home and Health Department.

\section{References}

1 Corsellis JAN. Mental Illness and the Ageing Brain. London: Oxford University Press, 1962.

2 Tomlinson BE, Blessed G, Roth M. Observations on the brains of demented old people. J Neurol Sci 1970; 11:205-42.

3 Gawler J, du Boulay GH, Bull JWD, Marshall J. Computerised tomography (the EMI scanner): a comparison with pneumoencephalography and ventriculography. J Neurol Neurosurg Psychiatry 1976; 39:203-11.

4 Roberts MA, Caird FI, Grossart KW, Steven JL. Computerised tomography in the diagnosis of cerebral atrophy. J Neurol Neurosurg Psychiatry 1976; 39:909-15.

5 Huckman MS, Fox J, Topel J. The validity of criteria for the evaluation of cerebral atrophy by computed tomography. Radiology 1975; 116: 85-92.

6 Marsden CD, Harrison MJG. Outcome of investigation of patients with presenile dementia. $\mathrm{Br}$ Med J 1972; 1:249-52.

7 Corsellis JAN. Ageing and the Dementias. In: Blackwood W, Corsellis JAN, ed. Greenfield's Neuropathology. London: Arnold 1976; 797-848.

8 Hubbard BM, Anderson JM. A quantitative study of cerebral atrophy in old age and senile dementia. J Neurol Sci 1981; 50:135-45.

9 Dunnill MS. Quantitative methods in the study of pulmonary pathology. Thorax 1962; 17:320-8.

10 Wyper DJ, Pickard JD, Matheson M. Accuracy of ventricular volume estimation. J Neurol Neurosurg Psychiatry 1979; 42:345-50.

11 Davis PJM, Wright EA. A new method for measuring cranial cavity volume and its appli- 
cation to the assessment of cerebral atrophy at autopsy. Neuropathol Appl Neurobiol 1977; 3: 341-58.

12 Ball MJ. Neurofibrillary tangles and the pathogenesis of dementia; a quantitative study. Neuropathol Appl Neurobiol 1976; 2:395-410.

13 Hirano A, Zimmerman HM. Alzheimer's neurofibrillary changes: a topographic study. Arch Neurol 1962; 7:227-42.

14 Dayan AD. Quantitative histological studies on the aged human brain. II. Senile plaques and neurofibrillary tangles in senile dementia. Acta Neuropathol (Berl) 1970; 16:95-102.

15 Sarwar M, McCormick WF. Decrease in ventricular and sulcal size after death. Radiology 1978; 127:409-11.

16 Synek V, Reuben JR. The ventricular-brain ratio using planimetric measurement of EMI scans. Br J Radiol 1976; 49:233-7.

17 Barron SA, Jacobs L, Kinkel WR. Changes in size of normal lateral ventricles during ageing determined by computerized tomography. Neurology (Minneap) 1976; 26:1011-3.

18 De Souza SW, Dobbing J. Cerebral edema in developing brain: I. Normal water and cation content in developing rat brain and postmortem changes. Exp Neurol 1971; 32:431-8.

19 Tomlinson BE, Blessed G, Roth M. Observations on the brains of non-demented old people. $J$ Neurol Sci 1968; 7:331-56.

20 Jacoby RJ, Levy R, Dawson JM. Computed tomography in the elderly. I. The normal population. Br J Psychiatry 1980; 136:249-55.

21 Gosling RH. The association of dementia with radiologically demonstrated cerebral atrophy. J Neurol Neurosurg Psychiatry 1955; 18:129-33.

22 Mutrux S. Contribution à l'étude des correlations anatomo-cliniques dans les démences dégénératives et les démences arterioscléreuses. Monatsschr Psychiatrie Neurol (Basel Schweiz) 1953; 125: 19-38.

23 Jacoby RJ, Levy R. Computed tomography in the elderly. II. Senile dementia: diagnosis and functional impairment. Br J Psychiatry 1980; 136: 256-69.

24 Roberts MA, Caird FI. Computerised tomography and intellectual impairment in the elderly. J Neurol Neurosurg Psychiatry 1976; 39:986-9. 